\title{
MULTIPLE CONDUCTION SYSTEMS AND THE BEHAVIOUR OF SEA ANEMONES
}

$\operatorname{AUTHOR}(\mathrm{S})$ :

Mcfarlane, I. D.

\section{CITATION:}

Mcfarlane, I. D.. MULTIPLE CONDUCTION SYSTEMS AND THE BEHAVIOUR OF SEA

ANEMONES. PUBLICATIONS OF THE SETO MARINE BIOLOGICAL LABORATORY 1973, 20: $513-523$

ISSUE DATE:

1973-12-19

URL:

http://hdl.handle.net/2433/175761

RIGHT: 


\title{
MULTIPLE CONDUCTION SYSTEMS AND THE BEHAVIOUR OF SEA ANEMONES
}

\author{
I. D. MCFARLANE \\ Gatty Marine Laboratory and Department of Zoology \\ University of St. Andrews, Fife, Scotland
}

With 3 Text-figures

\begin{abstract}
Electrical activity can be recorded from three distinct conduction systems-the through-conducting nerve net and two slow systems (SS1 and SS2). Spontaneous and evoked pulses are shown in recordings from Metridium senile. The responses and interactions of these systems seem to control important components of actinian behaviour. SS1 pulses are seen during the opening of Tealia felina in response to dissolved food substances and during shell-climbing in Calliactis parasitica. SS1 activity leads to relaxation of oral disk radial muscles in Tealia and to pedal disk detachment in Calliactis. The nerve net and SS2 may control contraction of endodermal muscles in Calliactis.
\end{abstract}

\section{Experimental Analysis of the Behaviour of Sea Anemones}

Recordings made with extracellular suction electrodes have provided evidence of multiple conduction systems in a number of hydrozoan, scyphozoan and anthozoan species. Research has been concentrated mainly on Hydra, hydromedusae, and colonial hydroids, where recorded pulses are often large, perhaps reaching several millivolts (e.g. Passano \& McCullough, 1963; Mackie \& Passano, 1968; Josephson, 1965). These large pulses may represent activity in non-nervous (neuroid or myoid) conduction systems, but conduction in the absence of nervous elements has been clearly established only in the hydromedusae (see review by MACKIE, 1970). Difficulty often occurs in relating activity of these conduction systems to behavioural events. Some of the large pulses occur spontaneously and appear to accompany activities such as tentacle concerts in Tubularia (JOSEPHSON \& MACKIE, 1965) and hydranth contraction in Obelia (MoRIN \& COOKE, 1971). However, as MACKIE (1968) points out for Cordylophora, pulses may occur in the absence of detectable behavioural events and conversely certain behavioural activities may occur in the absence of recordable electrical events.

Similar studies with sea anemones offer certain advantages:

1. The animals are large, solitary and sessile. 2. There is a considerable background of histological, physiological and behavioural knowledge, concentrated mainly on the species Calliactis parasitica, Metridium senile, and Stomphia coccinea. 3. Isolated 
preparations survive well and enable monitoring of events that are difficult to follow in the intact animal, such as spontaneous contractions of endodermal muscles. 4. Some species show complex behavioural sequences that are readily elicited by known stimuli. For instance, the shell-climbing behaviour of Calliactis parasitica provides an example of the combination of certain basic neuromuscular activities into a pattern that is amenable to analysis (Ross \& Surton, 1961 a). DAVEnPort (1966) has discussed, with particular reference to the relationship between Calliactis and its hermit crab partner, the advantages of using symbiotic animals for behavioural studies. The main difficulties in trying to relate behaviour to electrical activity of conduction systems in sea anemones are that recorded pulses tend to be very small (often only reaching a few microvolts) and that pulses can often only be clearly recorded from the tentacles and oral disk.

PANTIN's (1935) classic study of the neuromuscular physiology of Calliactis parasitica established the action of the through-conducting nerve net in co-ordinating rapid symmetrical contractions of the sphincter muscle. JOSEPHSON (1966) showed that suction electrodes record large muscle action potentials associated with fast contractions of the tentacles and sphincter of Calliactis polypus. Small, short duration pulses, believed to be action potentials from neurites in the nerve net, have been recorded from mesenteries of Metridium senile (ROBSON \& JOSEPHSON, 1969) and Calamactis praelongus (PICKENS, 1969). Similar pulses have been recorded from tentacles of Calliactis parasitica (MCFARLANE, 1969 a, 1972). Recordings show the presence of two additional conduction systems, the SS1 and SS2, in Calliactis parasitica (MCFARLANE, $1969 a$ ) and apparently equivalent systems in a number of other species (MCFARLANE, 1972). Both these systems are slowly-conducting in comparison to the nerve net, and have refractory periods many times greater than the through-conducting nerve net. It is not known whether the SS1 and SS2 are additional nerve nets or are non-nervous conduction systems. Fig. 1 shows spontaneous and evoked pulses from the three known conduction systems, nerve net, SS1 and SS2, recorded from tentacles of Metridium. Note that the SS1 and SS2 pulses are of much longer duration than the nerve net pulse and are apparently conducted at a much lower conduction velocity. The systems are distinct in that each shows a different threshold for electrical stimulation.

The nerve net pulse is seen following electrical stimulation of any part of Calliactis parasitica and is believed to represent a single impulse in the nerve net supplying the ectodermal musculature of the tentacles and oral disk. In Mimetridium cryptum there is histological evidence that this ectodermal nerve net is connected to the endodermal nerve net overlying the retractor muscles of the mesenteries (BATHAM, 1965). The pulse is thus taken as indicating activity in the through-conducting nerve net studied by PANTIN (1935). Nerves and muscles may be absent from column and pedal disk ectoderm, and yet the SS1 seems to extend throughout the entire ectoderm (McFarlane, 1969 a). The possibility that the SS1 is a non-nervous conduction system, perhaps involving communication between the supporting cells of the ectoderm, 


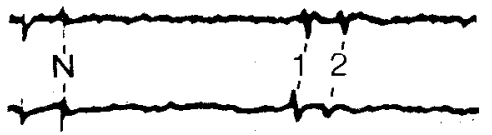

A

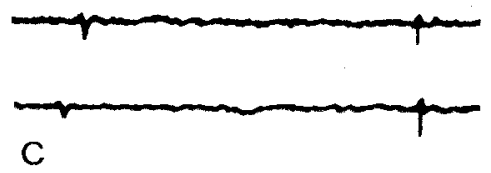

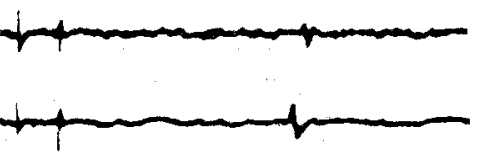

B

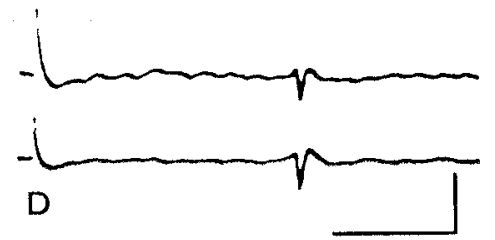

Fig. 1. Electrical activity recorded from the sea anemone Metridium senile. Two recording electrodes were used, attached to tentacles on opposite sides of the oral disk. A. Response to a single shock to base of column. Conduction path about $8 \mathrm{~cm}$. Temperature $14^{\circ} \mathrm{C}$. The stimulus artefact is followed by a nerve net pulse (N), SS1 pulse (1) and SS2 pulse (2). Note the long conduction delay for the slow systems. B. Stimulation in the same position but at a slightly lower stimulus intensity elicits nerve net and SS1 pulses only. At even lower intensity only the nerve net pulse follows stimulation. C. Spontaneous pulses-here a spontaneous SS2 pulse is followed by a nerve net pulse. D. A faster time base shows that the nerve net pulse following a single shock is of short duration (less than $20 \mathrm{msec}$.). Vertical scale $=10 \mu \mathrm{V}$. Horizontal scale $=500 \mathrm{msec}$. for A, B, C; $80 \mathrm{msec}$. for D.

cannot as yet be directly confirmed as the ectodermal cells are too small for intracellular recordings to be made. The SS2 seems to be endodermal and may involve myoid conduction between the cells making up the endodermal musculo-epithelium. Conduction may or may not involve associated contraction. However, there are endodermal nerve elements that may be functionally separate from the through-conducting nerve net (RoBSON, $1961 a, 1965)$ and may be the site of the SS2.

Although much previous physiological and histological information refers to Metridium senile, an analysis of the behaviour of this species in terms of known conduction systems is made difficult by the extreme small size of recorded pulses and the fact that SS1 and SS2 pulses are of similar appearance and difficult to distinguish. Most electrophysiological studies have involved Calliactis parasitica and Tealia felina, where recorded pulses are larger and more readily told apart (McFARLANE, $1969 a$, 1970). Examples of the types of behavioural analysis being attempted will illustrate the methods used.

\section{Preparatory Feeding Behaviour of Sea Anemones}

One of the earliest field observations made by naturalists studying sea anemones in rock pools was that the presence of dissolved food substances in the water will cause some species to open and become very expanded (e.g. Pollock, 1883). 
Allabach (1905) and Parker (1917) describe expansion of the oral disk of Metridium senile following exposure to mussel extract, but state that there was little change in column length. However, according to Pantin (1950) expansion is often followed by extension of the column and 'swaying'. This apparently purposeful activity may serve to increase the likelihood of contacting nearby food. PANTIN (1965) says that re-examination of earlier cine films suggests that the column bending movements ('swaying') are produced by local contraction of longitudinal parietal muscles.

This preparatory or pre-feeding response has been taken as indicating the presence of an olfactory sense in sea anemones. Tealia felina var. lofotensis, although a deepwater form, shows a similar pre-feeding response and when electrical activity is recorded from tentacles it is clear that the action of food extracts involves initiation of activity in the SS1 (MCFARLANE, 1970). The observed burst of SS1 pulses is followed by opening and expansion. Oral disk expansion also follows electrical stimulation of the SS1. The response could be clearly detected over a stimulus frequency range of 1 shock every $5 \mathrm{sec}$. to 1 shock every $30 \mathrm{sec}$. Only a small number of pulses seem

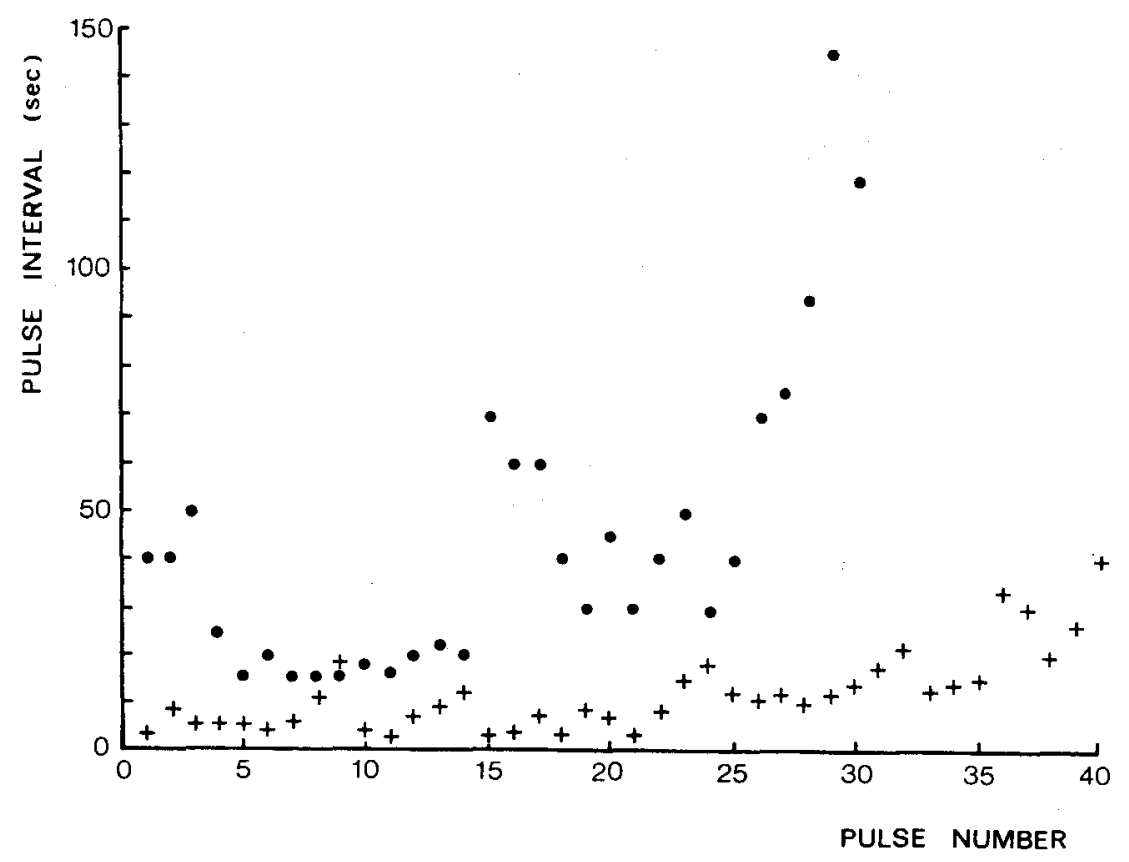

Fig. 2. SS1 sensory responses. A series of SS1 pulses follows application of mussel extract to the column of Tealia felina (see text for technique used). A single typical series is here plotted (-) as the interval between successive pulses against pulse number (e.g. in the case shown the first and second observed pulses were $40 \mathrm{sec}$. apart). As the response proceeds there is a gradual increase in pulse interval, possibly representing sensory adaptation. Similarly an example is given $(+)$ of the SS1 response when tentacles of Calliactis parasitica respond to a Buccinum shell. Note that the interval between pulses tends to be shorter in the Calliactis response. Data from McFarlane \& Lawn, 1972 and McFarlane, $1969 b$ ). 
required to start the response. The SS1 activity seems to cause relaxation of the ectodermal radial muscles of the oral disk (MCFARLANE \& LAWN, 1972). The location of the receptors or receptive surface concerned in the natural response is shown by the fact that a series of SS1 pulses follows attachment to the column, by suction, of a fine polythene tube containing food extract, but does not follow attachment of a tube containing sea water (MCFARLANE \& LAWN, 1972). The receptor response seems to show slow adaptation (Fig. 2).

The SS1 in Tealia felina is also known to respond to mechanical stimulation of the column, sensitivity being greatest near the base (MCFARLANE, 1970). It is therefore possible that the response to food extract involves another conduction system that elicits expansion, resulting in deformation of the column and mechanical stimulation of the SS1. However, the following points support the interpretation that this is a true chemosensory response of the SS1: 1. The first SS1 pulse may appear within a few seconds of application of the food extract, before any obvious oral disk expansion is seen. 2. Electrical stimulation of the SS1 elicits oral disk expansion. 3. LAWN (personal communication) has shown that all the observed SS1 pulses originate at the site of the applied chemical stimulus.

As in Metridium senile, the response may proceed further to column extension and bending. If SS1 activation is the only sensory response involved, a problem exists as to how the changes in activity of the endodermal muscles concerned in these movements are evoked by the SS1, a system that appears to be restricted to the ectoderm. As we shall see below, the same problem arises in the analysis of the shellclimbing behaviour of Calliactis parasitica.

\section{Shell-climbing Behaviour in Calliactis parasitica}

Only a very few SS1 pulses, at very low-frequency, are recorded from tentacles when Calliactis parasitica is exposed to mussel extract. It seems that the SS1 is primarily involved in a completely different sensory response in this species. The shellclimbing behaviour of Calliactis is well-known. Calliactis is normally found on Buccinum shells containing the hermit crab Pagurus bernhardus. Anemones fixed to other substrates can transfer their attachment to the shell by a sequence of movements involving the tentacles clinging to the shell, release of pedal disk attachment, swinging of the freed pedal disk round to the shell, and re-attachment. A major advance in our understanding of this behaviour came when Ross \& SutTon (1961 a) showed that the entire sequence is initiated by the tentacles responding to a so-far unidentified 'shell-factor'. Continued tentacle-shell contact is necessary for the sequence to proceed to the point of pedal disk re-attachment. Monitoring of electrical activity suggests that, as in the pre-feeding response of Tealia felina, SS1 activity is intimately concerned in the shell response. A series of SS1 pulses is recorded when tentacles respond to a shell and this continues only as long as tentacle-shell contact 
is maintained (MCFARLANE, $1969 \mathrm{~b}$ ). Electrical stimulation of the SS1 shows that the initial action of SS1 pulses is to cause detachment of the pedal disk (MCFARLANE, $1969 \mathrm{~b}$ ). Detachment is elicited by stimulation at frequencies between 1 shock every $3 \mathrm{sec}$. and 1 every $10 \mathrm{sec}$. (at $14^{\circ} \mathrm{C}$.). At all frequencies about 30 shocks were necessary to produce complete freeing of the pedal disk. In the tropical species Calliactis polypus electrical stimulation causes detachment and again the number of stimuli required is of the same order of magnitude at different stimulus frequencies (Ross \& SUTTON, 1970), but here the conduction system co-ordinating detachment has not yet been identified. Fig. 2 shows that in Calliactis parasitica the frequency of observed SS1 pulses is greater than 1 every 10 sec. during the early part of shell-climbing. In the case shown detachment took place at about pulse 24 in the series. The SS1 frequency seems to fall following detachment; this may result from slow sensory adaptation as seen in the pre-feeding response of Tealia felina.

The pedal disk of Calliactis parasitica is normally firmly attached by what appears to be a cement layer, yet during shell-climbing the pedal disk detaches cleanly, leaving the cement layer behind. Stomphia coccinea shows detachment and swimming following contact with certain species of starfish and nudibranch (YentsCH \& PIERCE, 1955; RobSON, 1961 b). ElLIS, Ross and SutTON (1969) conclude that detachment in Stomphia results from a combination of muscular action and chemical shedding of a cement layer. The same combination of actions may be responsible for detachment in Calliactis but as electrical stimulation of the SSI can cause detachment without any apparent associated muscular activity, it is possible that chemical destruction of cement is here a sufficient cause of loss of adhesion. This may not be true for Stomphia where detachment occurs more rapidly. During natural or electrically stimulated detachment of Calliactis it is often seen that the mucous layer that extends some way up the column, is shed as a single sheet. The same phenomenon has been reported for Stomphia (ElLIS, Ross \& SutTon, 1969). The implication is that the normal secretory products of the column have been modified in the pedal disk to produce an adhesive cement, but that both mucus and cement may be shed by the same process following SS1 activity. It would seem that in Calliactis this mucous coat could only be cast by chemical means as it is normally closely adhering to the surface of the column and because of its flexibility it readily follows body movements. Supporting evidence for SS1 involvement may be found in the many references in the literature to shedding of the mucous coat of the column during feeding in many species (e.g. Actinia equina-in Dalyell, 1848). If other species have similar receptor mechanisms to Tealia felina, then during feeding diffusible activators may reach the column, excite SS1 pulses, and these pulses may in some way effect shedding of mucus. It is of interest that MACKIE (1970) suggests that some neuroid conduction systems may be involved in co-ordination of responses not normally considered to be effector actions - such as control of secretion. Neuroid conduction occurs between ectodermal epithelial cells of the larva of the toad Xenopus and here one of the proposed effector actions is mucus secretion (RoBERTS, 1971). 
The SS1 in Calliactis parasitica is also sensitive to mechanical stimulation of the column. In other anemone/crab partnerships, involving different species of Calliactis, examples are known where transfer of attachment is not effected by the anemone but by the crab. The crab prods the column repeatedly and this leads to relaxation and detachment. The anemone is then placed on the shell or carapace (Cutress \& Ross, 1969; Cutress, Ross \& Sutton, 1970; Ross, 1970; Ross \& Sutton, 1961 b). In some cases it has been shown that electrical and mechanical stimuli can also produce relaxation and detachment (Ross \& SUTTON, 1970). Presumably an SS1 system is involved. Ross and SuTTON say that one of the most effective ways of detaching Calliactis polypus is to stroke the base of the column with a fine brush. Such stimulation is effective in exciting the SS1 in Calliactis parasitica (MCFARLANE, 1969 b).

The fact that the SS1 has been shown to be active in two apparently different behavioural responses, pre-feeding in Tealia and shell-climbing in Calliactis, may imply that there is some form of evolutionary link between the two types of behaviour. However, the important differences between the two responses should be borne in mind (Table 1).

Table 1. A comparison of the characteristics of the SS1 response involved in the pre-feeding response of Tealia and shell-climbing of Calliactis. The effective frequency range refers to responses elicited by electrical stimulation.

\begin{tabular}{|c|c|c|c|c|}
\hline . & Stimulus & $\begin{array}{l}\text { Receptive } \\
\text { area }\end{array}$ & Response & $\begin{array}{l}\text { Effective SS1 } \\
\text { frequency range }\end{array}$ \\
\hline Pre-feeding: & $\begin{array}{l}\text { Soluble food } \\
\text { substance }\end{array}$ & Column & Expansion & $1 / 5-1 / 30 \mathrm{sec}$ \\
\hline Shell-climbing: & $\begin{array}{l}\text { Insoluble } \\
\text { 'shell-factor' }\end{array}$ & Tentacles & Detachment & $1 / 3-1 / 10 \mathrm{sec}$ \\
\hline
\end{tabular}

Calliactis parasitica does show SS1 pulses when exposed to food extracts, but generally the frequency and number of these pulses is too low to cause detachment. Logic tells us that this must be so, otherwise the anemone would detach every time it fed. It has not yet been clearly demonstrated that the few pulses that do occur cause anything equivalent to a pre-feeding response. Such a response might be of advantage as SS1 pulses following contact of dissolved food substances could signal that food is nearby, for example while the hermit crab is feeding. Column extension and bending might then enable food to be acquired. However, as in Tealia pre-feeding, it is not known if the SS1 is the only conduction system involved in the shell-response and if so how this apparently ectodermal system excites changes in activity of the endodermal muscles responsible for column extension and bending. Bursts of nerve net activity have been recorded during shell-climbing (MCFARLANE, $1969 \mathrm{~b}$ ) but as described below, such bursts are also recorded from animals that are not climbing.

It would be surprising to find such a well-developed behaviour pattern directed 
towards gaining a foothold on the shell only for the anemone to loose its hold when feeding. It seems that the important safeguard against this happening is the number and frequency of SS1 pulses required to elicit detachment. The presence of only a few receptors for the food chemical, or sensory adaptation, could prevent detachment occuring during feeding. In fact, a few specimens kept in an aquarium for several months have been observed to detach from shells during feeding and even to show certain components of shell-climbing, such as column bending. Presumably under natural conditions the concentration of the active food substance never reaches a high enough level to produce the frequency of SS1 pulses needed to cause detachment.

Calliactis parasitica normally lives on shells occupied by hermit crabs and the anemone is presumably often mechanically stimulated as the crab walks around. However, in order to feed the anemone must remain open as much as possible. This is largely achieved thanks to the rapid relaxation characteristics of the muscles involved in the protective withdrawal reflex (PANTIN, 1935) but this may be aided by occasional mechanically-induced SS1 pulses. These may be at a high enough frequency to cause expansion but are clearly not at a high enough frequency to cause detachment. In the aquarium, Calliactis that are kept on shells containing hermit crabs often appear more expanded than those kept on empty shells.

PANTIN (1965) points out that the preparatory feeding response of Metridium, shell-climbing in Calliactis, and swimming in Stomphia have certain features in common; for example column extension and bending. Clearly it is of importance to determine if Stomphia shows sensory activation of an SS1 system in response to contact with the starfish and nudibranchs that excite swimming.

\section{Spontaneous Contractions of Parietal Muscles in Calliactis}

As in Metridium senile (Fig. 1), spontaneous nerve net and SS2 pulses are recorded from tentacles of Calliactis parasitica. A study of recordings of electrical activity from half-animal preparations has shown that there are regular bursts of nerve net activity (MCFARLANE, 1972). Following such a burst SS2 activity almost immediately increases to a maximum, followed by a slow decline in frequency of observed SS2 pulses. The subsequent nerve net burst occurs during a period of low-frequency SS2 activity. This cycle may be repeated regularly but periods of irregular activity are also observed.

Isolated preparations of various muscles from sea anemones often show rhythmic spontaneous contractions. From a study of such preparations BATHAM and PANTIN (1954) proposed that large contractions of parietal muscles in Metridium senile are caused by bursts of through-conducting nerve net activity. Simultaneous monitoring of electrical activity and contractions from similar preparations has allowed direct confirmation of this for Calliactis parasitica. The record shown in Fig. 3 is from a preparation containing two connected strips of parietal muscle, cut from opposite sides of the column. Bursts of nerve net activity $(A, B, C)$ are followed by large 


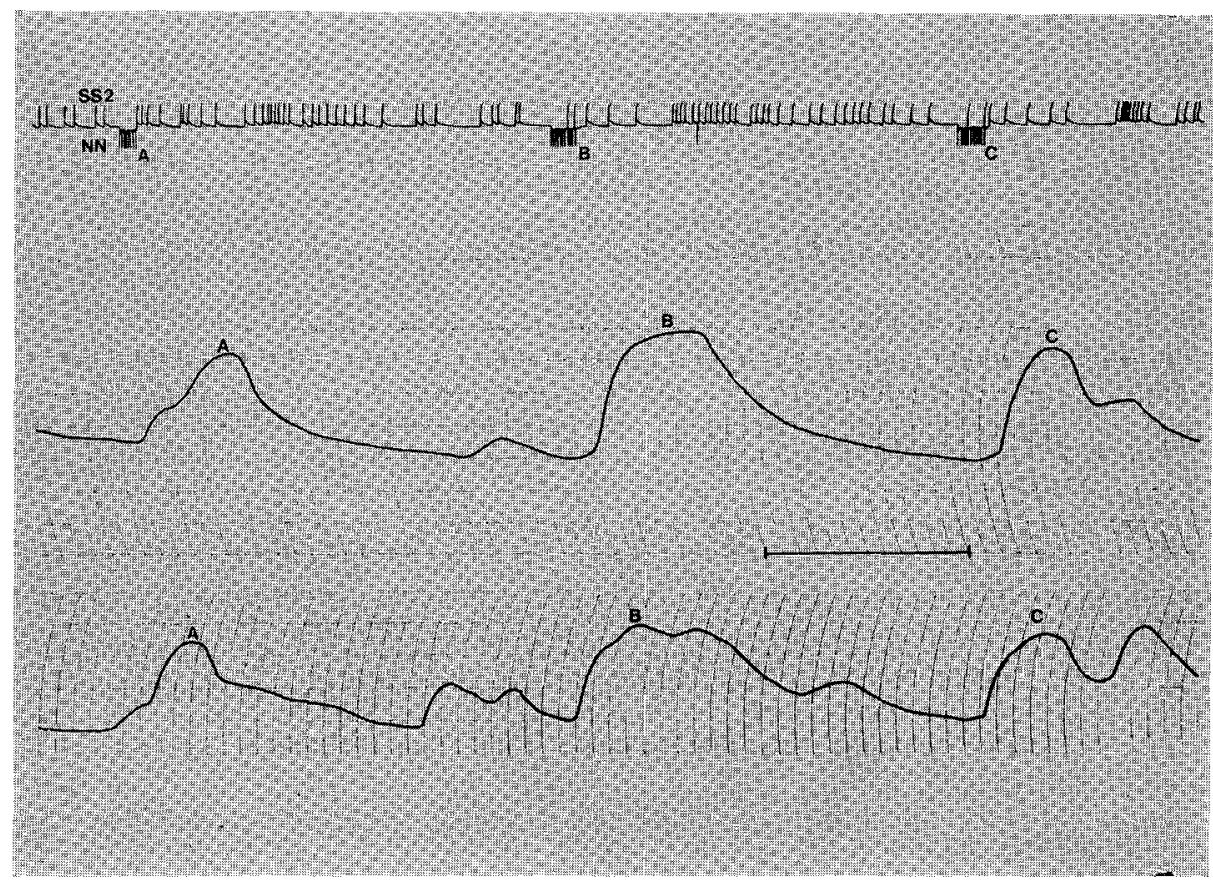

Fig. 3. Nerve net bursts and spontaneous contractions of parietal muscles in Calliactis parasitica. The preparation used enabled recording of parietal contractions from two connected strips of column, and simultaneous monitoring of nerve net (NN) and SS2 activity from tentacles. Electrical activity was displayed on a storage oscilloscope and observed pulses are marked on the upper trace (note that these are not actual recordings of pulses). Three nerve net bursts (A, B, C) are followed by large parietal contractions (A, B, C) in both parietal strips. Peak SS2 activity seems to occur during relaxation of the muscles. Note that small, apparently local, contractions can occur in the absence of recorded nerve net activity. Time scale $=5 \mathrm{~min}$. Temperature $=14^{\circ} \mathrm{C}$.

contractions of both parietal strips. The simplest interpretation is that the nerve net innervates the parietal muscles and the burst causes contraction. The strip shown in the lower record appears to start contracting about $25 \mathrm{sec}$. before the other strip; the reason for this is not known. The bursts here contain 8,11 , and 11 pulses respectively and the mean firing frequency in a burst is about 1 pulse every $3 \mathrm{sec}$. The interval between bursts is about $10 \mathrm{~min}$. Here, unlike in the half-animal preparations (MCFARLANe, 1972), there is a long delay between the nerve net burst and the subsequent increase in SS2 activity. This is seen most clearly following burst B. SS2 activity seems to be at a maximum just following the large parietal contractions. Although the SS2 rhythm seems in some way related to nerve net bursts and perhaps to the spontaneous contractions, the function of the SS2 is as yet not clear. An important point illustrated in Fig. 3 is that small, apparently local, contractions can occur in the absence of activity in the through-conducting nerve net. It should be remembered that electrical activity is being recorded from tentacles so only 'through- 
conducted' events are detected and any local electrical activity at the contracting regions is not recorded.

\section{REFERENCES}

Allabach, L. F. 1905. Some points regarding the behavior of Metridium. Biol. Bull. 10, $35-43$.

Batham, E. J. 1965. The neural architecture of the sea anemone Mimetridum cryptum. Am. Zool. $5,395-402$.

Batham, E. J. \& Pantin, C. F. A. 1954. Slow contraction and its relation to spontaneous activity in the sea-anemone Metridium senile (L.). J. exp. Biol. 31, 84-103.

Cutress, C. E. \& Ross, D. M. 1969. The sea anemone Calliactis tricolor and its association with the hermit crab Dardanus venosus. J. Zool. Lond. 158, 225-241.

Cutress, C. E., Ross, D. M. \& Sutton, L. 1970. The association of Calliactis tricolor wiith its pagurid, calappid, and majid partners in the Caribbean. Can. J. Zool. 48, 371-376.

Dalyell, J. G. 1848. Rare and Remarkable Animals of Scotland. 2. John van Voorst, London.

DavenPoRT, D. 1966. Cnidarian symbioses and the experimental analysis of behavior. Symp. zool. Soc. Lond. 16, 361-371.

Ellis, V. L., Ross, D. M. \& Sutton, L. 1969. The pedal disk of the swimming sea anemone Stomphia coccinea during detachment, swimming and resettlement. Can. J. Zool. 47, 333-342.

Josephson, R. K. 1965. Three parallel conducting systems in the stalk of a hydroid. J. exp. Biol. $42,139-152$.

JOSEPHSON, R. K. 1966. Neuromuscular transmission in a sea anemone. J. exp. Biol. 45, 305-320.

Josephson, R. K. \& MACKiE, G. O. 1965. Multiple pacemakers and the behaviour of the hydroid Tubularia. J. exp. Biol. 43, 293-332.

MCFarlane, I. D. 1969 a. Two slow conduction systems in the sea anemone Calliactis parasitica. J. exp. Biol. 51, 377-385.

McFarlane, I. D. $1969 \mathrm{~b}$. Co-ordination of pedal-disk detachment in the sea anemone Calliactis parasitica. J.exp. Biol. 51, 387-396.

MCFarlane, I. D. 1970. Control of preparatory feeding behaviour in the sea anemone Tealia felina. J. exp. Biol. 53, 211-220.

McFarlane, I. D. 1972. Spontaneous electrical activity in the sea anemone Calliactis parasitica. J. exp. Biol. 58, 77-90.

MCFARlane, I. D. \& LAWN, I. D. 1972. Expansion and contraction of the oral disk in the sea anemone Tealia felina. J. exp. Biol. 57, 633-649.

MACKIE, G. O. 1968. Electrical activity in the hydroid Cordylophora. J. exp. Biol. 49, 387-400.

MaCkIE, G. O. 1970. Neuroid conduction and the evolution of conducting tissues. Quart. Rev. Biol. 45, 319-332.

Mackie, G. O. \& Passano, L. M. 1968. Epithelial conduction in Hydromedusae. J. gen. Physiol. $52,600-621$.

Morin, J. G. \& Cooke, I. M. 1971. Behavioral physiology of the colonial hydroid obelia. 1. Spontaneous movements and correlated electrical activity. J. exp. Biol. 54, 689-706.

Pantin, C. F. A. 1935. The nerve-net of the Actinozoa. I. Facilitation. J. exp. Biol. 12, 119-138.

Pantin, C. F. A. 1950. Behaviour patterns in lower invertebrates. Symp. Soc. exp. Biol. 4, 175-195.

Pantin, C. F. A. 1965. Capabilities of the coelenterate behavior machine. Am. Zool. 5, 581-589.

Parker, G. H. 1917. Actinian behavior. J. exp. Zool. 22, 193-229.

Passano, L. M. \& McCullough, C. B. 1963. Pacemaker hierarchies controlling the behaviour of hydras. Nature, Lond. 199, 1174-5.

Pickens, P. E. 1969. Rapid contractions and associated potentials in a sand-dwelling anemone. J. exp. Biol.51, 513-528.

Pollock, W. H. 1883. On indications of the sense of smell in Actiniae. With an addendum by G. J. 
Romanes, J. Linn. Soc. Zool. 16, 474-476.

Roberts, A. 1971. The role of propagated skin impulses in the sensory system of young tadpoles. Z. vergl. Physiol. 75, 388-401.

Robson, E. A. 1961 a. A comparison of the nervous systems of two sea-anemones, Calliactis parasitica and Metridium senile. Q. J. microsc. Sci. 102, 319-326.

Robson, E. A. $1961 \mathrm{~b}$. Some observations on the swimming behaviour of the anemone Stomphia coccinea. J. exp. Biol. 38, 343-363.

Robson, E. A. \& Josephson, R. K. 1969. Neuromuscular properties of mesenteries from the seaanemone Metridium. J. exp. Biol. 50, 151-168.

Ross, D. M. 1970. The commensal association of Calliactis polypus and the hermit crab Dardanus gemmatus in Hawaii. Can. J. Zool. 48, 351-357.

Ross, D. M. \& SutTon, L. 1961 a. The response of the sea anemone Calliactis parasitica to shells of the hermit crab Pagurus bernhardus. Proc. R. Soc. B 155, 266-281.

Ross, D. M. \& Sutton, L. 1961 b. The association between the hermit crab Dardanus arrosor (Herbst) and the sea anemone Calliactis parasitica (Couch). Proc. R. Soc. B 155, 282-291.

Ross, D. M. \& Sutton, L. 1970. The detachment of the commensal sea anemones, Calliactis polypus and C. tricolor by mechanical and electrical stimulation. Z. vergl. Physiol. 67, 102-119.

Yentsch, C. S., \& Pierce, D. C. 1955. A 'swimming' anemone from Puget Sound. Science. 122, 1231-1233. 